\title{
Cue Duration Affects Attentional Capture without Modulating Inhibition of Return
}

\author{
Yukihisa Matsuda, Syoichi Iwasaki \\ Graduate School of Information Sciences, Tohoku University, Sendai, Japan \\ Email: matsuda-papers@hotmail.co.jp
}

Received July $20^{\text {th }}, 2012$; revised August $22^{\text {nd }}, 2012$; accepted September $15^{\text {th }}, 2012$

\begin{abstract}
In the cueing paradigm, an abrupt onset of the cue brings about both the facilitation effect and inhibition of return (IOR) depending on the cue-target interval. Previous studies showed that physical properties of the cue such as duration affect the occurrences of facilitation effect and IOR. However, other study indicated that cue duration did not affect these two effects. The first aim of this study was to clarify how cue duration affects the facilitation effect. The results showed that the temporal properties of the cue influenced the facilitation effect. The second aim of this study was to examine the relationship between the magnitude of the facilitation effect and that of IOR with the results in Experiments 1 and 2. There were four findings that suggested discrepancies in the effect of spatial cueing between the facilitation effect and IOR. In conclusion, these two processes were driven by distinct mechanisms.
\end{abstract}

Keywords: Attentional Mechanism; Attentional Capture; Inhibition of Return; Cue Duration; Brightness Change

\section{Introduction}

Visual attention enables one to select specific information in the visual scene. Since Posner developed the spatial cueing paradigm (Posner, 1978, 1980), two phenomena concerning bottom-up (or exogenous) attention have been well documented. In the cueing paradigm, two peripheral boxes (placeholders) are presented with one box for each visual field near the fixation point. An abrupt change in luminance (usually its increase) occurs as the cue. After the cue presentation, a target is presented at one of the two peripheral boxes. Trials in which the cue and the target appeared at the same location are classified as valid trials, and those in which they appeared at different locations (e.g., the cue is presented at the left box and target is presented at the right box or vice versa) are classified as invalid trials. Usually target detection reaction time (RT) is the dependent measure. RTs for detecting target are shorter in the valid condition than those for the invalid condition, because target processing is facilitated by attention captured by the cue. If the time interval between the onset of the cue and the onset of the target (stimulus onset asynchrony; SOA) is greater than approximately $250 \mathrm{~ms}$, the results are reversed: RTs in the valid condition are longer than those in the invalid condition. The former is called attentional capture (or facilitation), and the latter is called inhibition of return (IOR; Posner, \& Cohen, 1984; Posner, Rafal, Choate, \& Vaughan, 1985; Taylor \& Klein, 1998; Klein, 2000).

Previous studies have shown that physical properties of the cue, such as brightness (Wright \& Richard, 2003), spatial position (Pratt, Hillis, \& Gold, 2001; McAuliffe \& Pratt, 2005), degree of eccentricity (O'donnell \& Pratt, 1996; Berger, Dori, \& Henik, 1999) and duration (Berger et al., 1999; Maruff, Yucel, Danckert, Stuart, \& Currie, 1999; Collie, Maruff, Yuchel, Danckert, \& Currie, 2000; McAuliffe \& Pratt, 2005) affect the occurrences of attentional capture and IOR, and also their mag- nitudes. Concerning cue duration, the studies conducted by Maruff et al. (1999) and Collie et al. (2000) suggested that timing of the cue relative to the target presentation influenced occurrence and the magnitude of the facilitation effect. If the target was presented before the cue offset (overlap cue condition), the facilitation was observed at the $150 \mathrm{~ms} \mathrm{SOA}$. By contrast, if the target was presented after the cue offset (non-overlap cue condition), the facilitation effect was not observed even when SOA was $150 \mathrm{~ms}$. Because many studies that have found the facilitation effect used the overlap cue (i.e., Posner \& Cohen, 1984; Maylor, 1985; Rafal, Calabresi, Brennan, \& Sciolto, 1989), Maruff et al. (1999) and Collie et al. (2000) concluded that occurrence and the magnitude of the facilitation effect were affected by the temporal overlap of the two stimuli. Contrary to their conclusion, however, Berger et al. (1999) have shown that the magnitude of facilitation in the overlap cue condition was no different in magnitude from that of the non-overlap cue condition. Berger et al. (1999) compared the cue duration of 200 $\mathrm{ms}$ (thus the cue overlapping with the target) with that of 100 $\mathrm{ms}$ (non-overlap cue) with the same SOA of $150 \mathrm{~ms}$ and found that these two cue durations produced comparable size of facilitation. Thus, the question whether the cue duration could modulate the facilitation effect still seems to remain open.

This study was designed to gain understanding of how cue duration affects the facilitation and IOR. Although, Maruff et al. (1999) and Collie et al. (2000) examined the effect of cue duration on the facilitation effect in the simple detection task, there were methodological problem in their studies. Maruff et al. (1999) and Collie et al. (2000) used green circular placeholders and a red cue. The red cue was wider than the green placeholder and extinguished at the end of the cue presentation time. Therefore the cue in their studies appeared as a new object with an abrupt onset, which was suggested to be more powerful in capturing attention (Yantis \& Jonides, 1984; Jonides \& Yantis, 1988). On the other hand, Berger et al. (1999) used a more 
conventional cueing method with two boxes presented as placeholders and brightness change as the cue. Thus, it is possible that these methodological discrepancies might have led to the contradictory results. Following more conventional procedures of the spatial cueing method used in the majority of the spatial cueing studies, two square boxes were used as placeholders and brightening of one of these boxes was defined as the cue in this study.

The second aim of this study was to examine the relationship between the magnitude of the facilitation effect and that of IOR. Since the suggestion by Posner and Cohen (1984) that IOR is a result of attention being directed to the location a moment before, it is generally believed that a single attentional mechanism is responsible for these two phenomena. Maylor (1985) has showed such an association between the facilitation effect and IOR using a double cueing paradigm. In this paradigm, the cue is presented at two locations of four peripheral boxes. Maylor (1985) confirmed that the double cue produced half the magnitude of the facilitation effect as compared with that produced by a single cue at a short SOA. The double cues also halved the magnitude of IOR for a longer SOA. These results, to which Berlucchi (2006) referred to as the "forgotten" double-cueing experiments, constitute strong evidence for the association between IOR and attentional mechanism. Reuter-Lorenz, Jha, and Rosenquist (1996) also demonstrated that IOR was dependent on attentional facilitation. Focusing on target modality and target intensity, the cue properties that were well known to influence the magnitude of the facilitation effect, they showed that at longer SOAs both of these properties affected the magnitude of IOR alike. Thus they concluded that IOR was produced by the attentional mechanism that controlled attentional facilitation.

In contrast to these studies, there are some other studies that have demonstrated that the facilitation effect and IOR are dissociable. Maylor (1985) demonstrated it with temporal order judgment (TOJ). In TOJ, two targets were presented in short succession at two placeholders after the cue was presented with various SOAs. The participants required to judge the order of the two targets. Even when two targets were presented simultaneously, the target at the cued location was perceived to be the first of the two in the shorter SOA. Contrary to the faster perception at a short SOA, which was due to the attentional facilitation, the cued target was not judged to be delayed for the long SOA at which IOR would have been obtained with the detection task (see also Gibson \& Egeth, 1994). Collie et al. (2000) compared spatial distributions of the facilitation and IOR. In their study, five placeholders were used, one at the fixation point, other four at $9^{\circ}$ and $18^{\circ}$ from the fixation point, two for each visual field. When the cue was presented at $18^{\circ}$, the facilitation effect was also found at the $9^{\circ}$, whereas there was no IOR at this location. This result indicated that the attentional spotlight had covered a wider area around the cued spot, while IOR was limited to the area within the placeholder, suggesting that these two attentional effects reflected different manners in which attention operated on the processing of target.

Here, we focused on the relationship between the magnitude of the facilitation effect and IOR by comparing these across participants with the conventional spatial cueing task. The basic design of the experiment was identical with that adopted by Reuter-Lorenz et al. (1996). If two phenomena were driven by one and the same mechanism, those participants who show larger facilitation at the short SOA would also be those who show larger IOR at the long SOA and vice versa. Contrarily, if the two phenomena were driven by separable mechanisms, there would be no relationship (correlations) between the magnitude of the facilitation effect and IOR. The modulation of target brightness as used by Reuter-Lorenz et al. (1996) might have affected the two attentional effects in the opposite direction; increased brightness of the target would increase its power of attentional capture, while counteracting the IOR effect of the cue since attention was more powerfully attracted to the brighter target. The important point in the cueing paradigm is that how (much) attention is captured by the cue, which then affects the information processing of the target through attention summoned by the cue. It may be argued that the experimental procedure used by Reuter-Lorenz et al. (1996) caused attention to be controlled by the bright target, rather than by the cue, which may have confounded their results. Thus, we manipulated a cue property (i.e., its duration) and then compared RTs to the target that was kept to be identical across the cue conditions. This constitutes a more direct test of the independence of the facilitation effect and IOR because the attention to the cue was manipulated by the cue durations, while attention to the target was kept to be the same across the conditions.

\section{Experiment 1}

The aim of Experiment 1 was to examine that how cue duration affects the facilitation effect and IOR. Here, cue was defined as brightness changing as majority of the spatial cueing studies. If properties of the cue presenting made different results between Maruff et al. (1999), Collie et al. (2000) and Berger et al. (1999), the results would be identical with Berger et al. (1999), in this study.

\section{Method}

Participants. Sixteen participants (mean age $19.3 \mathrm{yr} ; 9$ males) with normal or corrected-to-normal vision participated in the study. All participants were naive as to the pur-pose of the experiment.

Apparatus and stimuli. The stimuli were presented on a 19-in. color screen monitor. Presentation of stimuli was performed with an Intel Pentium 4 computer. A fixation cross (“+”; $0.5^{\circ}$ ) that was at the center of screen and two peripheral boxes $\left(1.8^{\circ}\right.$ in height and width) located $9.3^{\circ}$ to the left and right of the fixation cross were presented on a black background. The fixation cross and two boxes had a luminance of $4.3 \mathrm{~cd} / \mathrm{m}^{2}$. The spatial cue consisted of a luminance increment of $4.3 \mathrm{~cd} / \mathrm{m}^{2}$ to $43.8 \mathrm{~cd} / \mathrm{m}^{2}$. The target was a small filled square $\left(0.2^{\circ}\right)$ which appeared in the center of one of the two peripheral boxes. The luminance of the target was $43.8 \mathrm{~cd} / \mathrm{m}^{2}$.

Procedure. The experiment took place in a dimly lit and sound-attenuated room. The participants were seated facing a computer monitor at the distance of $63 \mathrm{~cm}$. Their heads were stabilized with an adjustable chin-rest, and the computer keyboard was placed in front of the participants. Each trial started with the presentation of the fixation cross and two peripheral boxes against black background (Figure 1). A short warning tone was presented at the beginning of the trial and was followed by the fixation cross and two peripheral boxes. They were presented for $1500 \mathrm{~ms}$, after which the peripheral cue was presented for 30 or $300 \mathrm{~ms}$ at either the left or right peripheral 


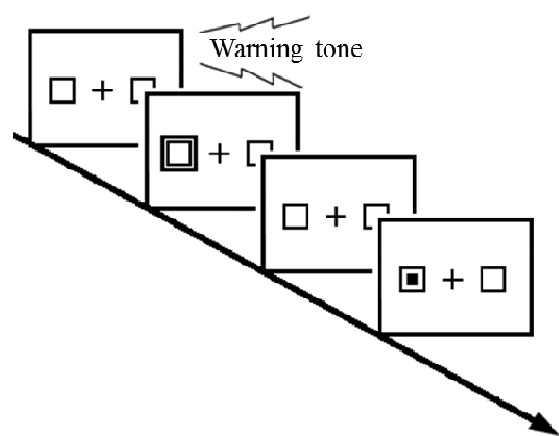

(a)

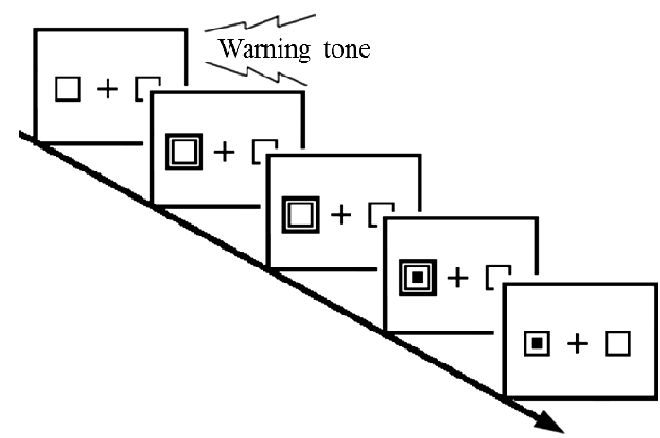

(b)

Figure 1.

Illustrations of the trial sequence used in Experiments 1 and 2. The panel (a) is the illustration of the non-overlap condition. The panel (b) is the illustration of the overlap condition. If SOA was $150 \mathrm{~ms}$ and cue duration was 200 or $300 \mathrm{~ms}$, cue and the target were overlapped while 50 or $150 \mathrm{~ms}$. The target was presented until response.

box. These two cue duration conditions were run in separated blocks. The SOA between the cue and the target were randomly selected among 150,450, and $800 \mathrm{~ms}$. The target could appear at either the left or right peripheral box after the peripheral cue was presented. The target remained on the screen until the key was pressed or $1500 \mathrm{~ms}$ had elapsed. A short tone was presented as a feedback signal for key pressing. The intertrial interval was $1500 \mathrm{~ms}$. Participants were instructed to maintain fixation on the fixation cross during a trial, ignoring the onset of the peripheral cue and to press the space bar with their right hand as quickly as possible when the target appeared. RT was measured from the onset of the target to response execution. Forty percent of all the trials were valid trials and another $40 \%$ were invalid trials. The remaining trials $(20 \%)$ were catch trials in which no target appeared. If participants responded during a catch trial, a beep one was presented as a warning. A practice block of 24 trials was run before the experimental blocks. There were two experiment blocks, one for each cue duration condition, with the total number of trials of 432 trials. The order of the blocks was randomized across participants. Short rests were given after 108 trials, and a longer rest was inserted between the blocks.

Analyses. Three-way mixed analyses of variance (ANOVA) were conducted for RT. Effect sizes for analysis of variance were calculated as partial eta-squared $\left(\eta_{\mathrm{p}}^{2}\right)$ and for $t$ tests, Cohen's $d$. Follow-up $t$ tests were performed when interactions were significant.

\section{Results}

The RTs either over $1000 \mathrm{~ms}$ or less than $200 \mathrm{~ms}$ were discarded from the data analysis. Standard deviation (SD) was obtained in each condition, and then the RTs over 2.5 SD of the mean of each condition were also discarded. Overall, $2.48 \%$ of the data were removed on average across all participants.

RTs. Figure 2 showed the results. Analysis for RTs, a threeway mixed ANOVA was performed with cue duration (30 and $300 \mathrm{~ms}) \times$ trial type (valid and invalid) $\times \operatorname{SOA}(150,450$, and $800 \mathrm{~ms}$ ) as within-group factors. The main effect of cue duration was not significant $\left[F(1,15)=0.49, p>.44, \eta_{p}^{2}=0.003\right]$. The main effect of SOA was significant $[F(1,15)=87.74, p$ $\left.<.001, \eta_{\mathrm{p}}^{2}=0.85\right]$. The main effect of trial type was significant $\left[F(1,15)=20.18, p<.001, \eta_{\mathrm{p}}^{2}=0.57\right]$. The two-way interaction between cue duration and SOA was significant $[F(2$, $\left.30)=5.09, p<.05, \eta_{\mathrm{p}}^{2}=0.25\right]$. The two-way interaction between cue duration and trial type was not significant $[F(1,15)=$ $\left.0.001, p>.97, \eta_{\mathrm{p}}^{2}<0.001\right]$. The two-way interaction between SOA and trial type was significant $[F(2,30)=26.25, p<0.001$, $\left.\eta_{\mathrm{p}}^{2}=0.64\right]$. The three-way interaction was significant $[F(2,30)$ $\left.=4.09, p<0.05, \eta_{\mathrm{p}}^{2}=0.21\right]$.

Our main interest was in comparing valid RTs and invalid RTs in each SOA and cue duration condition. A significant difference between valid and invalid RTs was the evidence that the facilitation effect or IOR was found depending on the direction of the RT difference. Simple effects of the three-way interaction indicated that difference between valid RTs and invalid RTs did not reach a significance level for the $30 \mathrm{~ms}$ cue duration, but it was significant for the $300 \mathrm{~ms}$ cue duration in the $150 \mathrm{~ms} \operatorname{SOA}\left[F(1,90)=7.26, p<0.001, \eta_{\mathrm{p}}^{2}=0.08\right]$. These results indicated that the overlap cue induced the facilitation effect but the non-overlap cue did not. In the $450 \mathrm{~ms}$ SOA, the differences between valid RTs and invalid RTs reached a significant level in the $30 \mathrm{~ms}$ cue duration $[F(1,90)=18.70, p<$ $\left.0.001, \eta_{\mathrm{p}}^{2}=0.17\right]$, and in the $300 \mathrm{~ms}$ cue duration $[F(1,90)=$ 19.37, $\left.p<0.001, \eta_{\mathrm{p}}^{2}=0.18\right]$. In the $800 \mathrm{~ms}$ SOA, difference between valid RTs and invalid RTs reached a significant level in the $30 \mathrm{~ms}$ cue duration $\left[F(1,90)=21.15, p<0.001, \eta_{\mathrm{p}}^{2}=\right.$ $0.19]$, and in the $300 \mathrm{~ms}$ cue duration $[F(1,90)=44.15, p<$ $\left.0.001, \eta_{\mathrm{p}}^{2}=0.33\right]$. As can be seen from Figure 2, the latter two differences were IORs with slower RTs for the valid conditions relative to the invalid conditions.

\section{Discussion}

The results showed that the overlap cue (300 ms cue duration) led to a significant facilitation effect with faster RTs for the valid condition than those for the invalid condition in the 150 ms SOA. In contrast, there was no difference in RTs be-tween the valid and invalid conditions for the non-overlap cue $(30 \mathrm{~ms}$ cue duration). Thus, the facilitation effect was differentially affected by the cue duration. In the 450 and $800 \mathrm{~ms}$ SOA when IORs were observed, there were significant differences between valid RTs and invalid RTs for both cue durations.

Unlike the facilitation effect that was found only with the overlap cue in the $150 \mathrm{~ms}$ SOA, IOR was not affected by the temporal property of the cue. This discrepancy may be a piece of evidence that there were separate underlying mechanisms for the facilitation effect and IOR. 
$30 \mathrm{~ms}$ cue condition

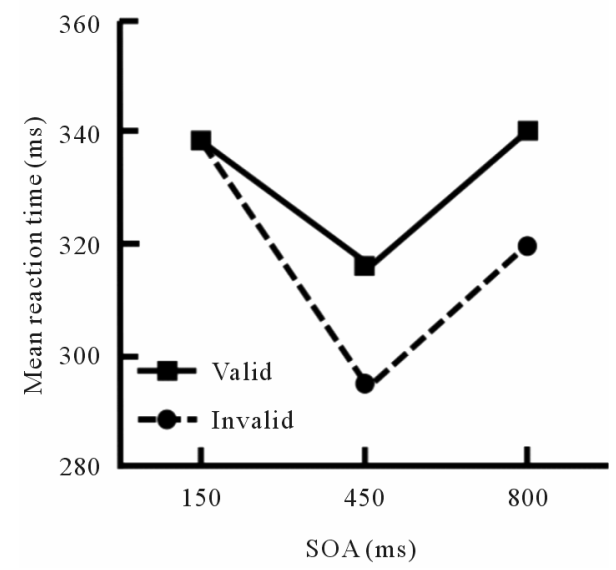

(a)

$300 \mathrm{~ms}$ cue condition

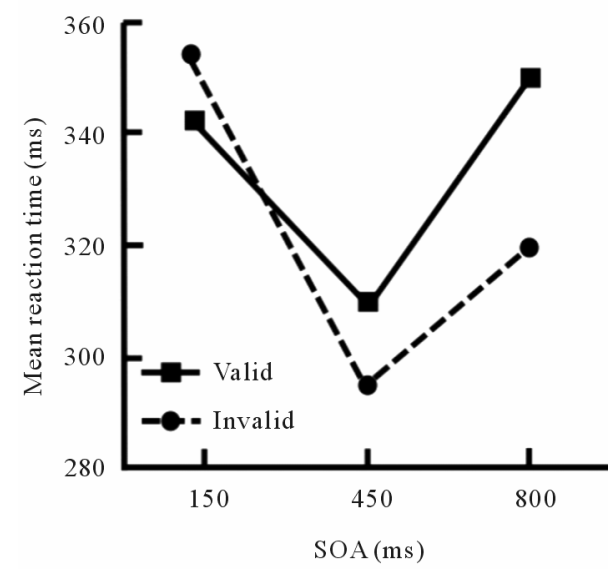

(b)

\section{Figure 2.}

Mean reaction times as a function of SOA for valid RTs (solid lines) and invalid RTs (dashed lines). The panel (a) is the illustration of the $30 \mathrm{~ms}$ cue duration condition (non-overlap cue). The panel (b) is the illustration of the $300 \mathrm{~ms}$ cue duration condition (overlap cue).

\section{Experiment 2}

In Experiment 1, both overlap and non-overlap cues were used. Although the temporal property of the cue affected whether attentional facilitation was observed or not, it is not clear whether this facilitation was caused by the presence of the cue (remaining visible or disappeared when the target was presented) or durations of the cue was critical in producing the results. To examine which factor was critical for the presence of the facilitation effect shown in Experiment 1, four cue durations (50, 100, 200, and $300 \mathrm{~ms}$ ) and two SOAs (150 and 450 $\mathrm{ms}$ ) were used in Experiment 2. If presence of the cue were critical, neither 50 nor $100 \mathrm{~ms}$ cues would show the facilitation effect in the $150 \mathrm{~ms}$ SOA, whereas the cue duration was the critical factor then the facilitation effect would increase with the cue duration.

The aim of Experiment 2 was to examine the relationship between the facilitation effect and IOR. We examined the relationship between the facilitation effect and IOR with two analyses. First, correlations between the facilitation effect and IOR were calculated. If IOR and the facilitation effect were controlled by the same attentional mechanism, there should be negative correlations between these two attentional phenomena (Notably, the magnitude of facilitation effect and IOR were calculated by invalid RTs minus valid RTs. Thus, large negative number indicates large magnitude of IOR, and large positive number indicates large magnitude of facilitation effect). The correlations were calculated separately for each cue duration condition. Second, we compared the magnitude of IOR between the non-overlap cue condition and the overlap cue condition (see correlations between the facilitation effect and IOR, and also comparisons of IOR with the overlap cue and the non-overlap cue in the results). If IOR and the facilitation effect are dominated by the same attentional mechanism, a manipulation that affects one effect of attention (e.g., facilitation) to some extent should affect the other (e.g., IOR) as well to the same extent. For this comparison, we combined the data of 15 participants from Experiment 1 and those of 16 participants from Experiment 2 to increase the size of the data pool for each condition. The RTs in the $50 \mathrm{~ms}$ cue duration condition of Experiment 1 and those of the $30 \mathrm{~ms}$ cue duration condition of Experiment 2 were combined as the non-overlap cue condition. The RTs in the $300 \mathrm{~ms}$ cue duration condition of Experiments 1 and 2 were combined as the overlap cue condition. Third, we compared IORs of the two groups formed by the median split based on the facilitation (see comparisons of two IOR groups formed by the magnitude of facilitation in the results). If the same attentional mechanism is underlying the facilitation effect and IOR, it would be expected that participants who showed larger facilitation should also show larger IOR, and those whose facilitation was smaller would show smaller IOR.

\section{Method}

Participants. Fifteen participants (mean age 18.9 yr; 8 males) with normal or corrected-to-normal vision participated in the study. All participants were naïve as to the purpose of the experiment.

Procedure. Procedure and materials were the same in experiment 1, with the following exceptions. First, there were four cue durations $(50,100,200$ and $300 \mathrm{~ms})$. Second, there were two SOAs, of 150 and $450 \mathrm{~ms}$. A practice block of 24 trials was run before the experimental blocks. There were four experiment blocks, which consisted of four cue duration conditions with a total of 480 trials. The SOAs were randomized within a block. The order of the blocks was randomized across participants. A short rest was inserted after 60 trials, and longer rests were given every two blocks.

\section{Results}

The RTs either over $1000 \mathrm{~ms}$ or less than $200 \mathrm{~ms}$ were discarded from the data analysis. After this elimination procedure SD was obtained for each condition. Then the RTs outside of 2.5 SD of the mean of each condition were further removed. Overall, $6.02 \%$ of the data were removed on the average across all participants.

RTs. Figure 3 showed the results. A three-way mixed ANOVA was performed on RTs with cue duration (50, 100, 


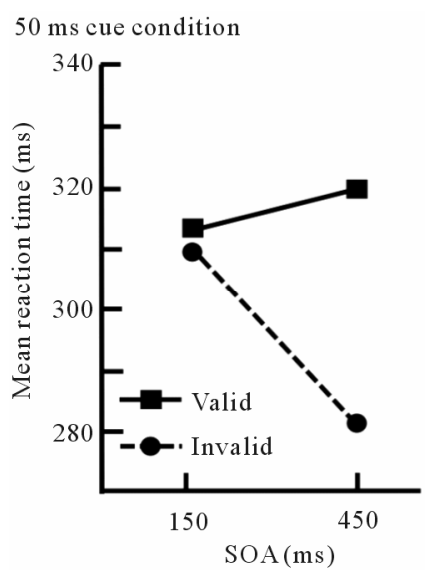

(a)

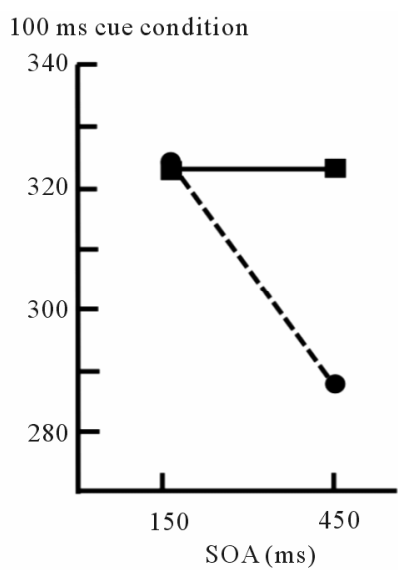

(b)

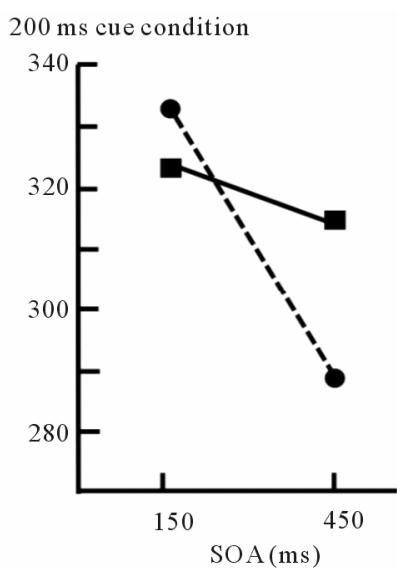

(c)

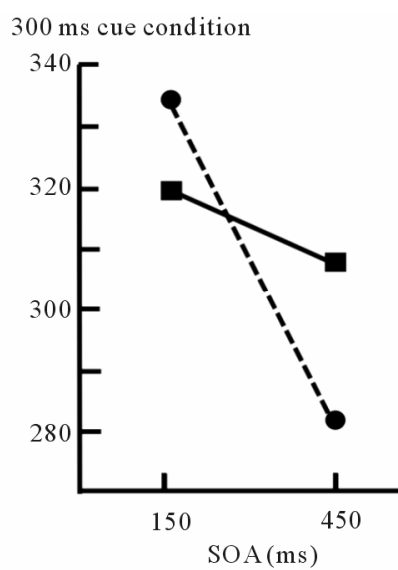

(d)

Figure 3.

Mean reaction time (ms) as a function of SOA for valid RTs (solid lines) and invalid RTs (dashed lines).

200 , and $300 \mathrm{~ms}) \times$ trial type (valid and invalid) $\times$ SOA $(150$ and $450 \mathrm{~ms})$ as within-group factors. The main effect of cue duration was not significant $\left[F(3,42)=0.82, p>0.48, \eta_{\mathrm{p}}^{2}=\right.$ $0.06]$. The main effect of SOA was significant $[F(1,14)=$ $\left.62.63, p<0.001, \eta_{p}^{2}=0.82\right]$. The main effect of trial type was significant $\left[F(1,14)=17.57, p<.001, \eta_{\mathrm{p}}^{2}=0.56\right]$. The twoway interaction between cue duration and SOA was significant $\left[F(3,42)=8.65, p<0.001, \eta_{\mathrm{p}}^{2}=0.38\right]$. The two-way interaction between cue duration and trial type was significant $[F(3$, $\left.42)=8.95, p<0.001, \eta_{\mathrm{p}}^{2}=0.39\right]$. The two-way interaction between SOA and trial type was significant $[F(1,14)=119.95$, $\left.p<0.001, \eta_{\mathrm{p}}^{2}=0.90\right]$. The three-way interaction was not significant $\left[F(3,42)=0.15, p>0.93, \eta_{\mathrm{p}}^{2}=0.01\right]$.

Although no significant three-way interaction was found, we conducted analyses of the simple effects since our main interest was to compare valid and invalid RTs for each SOA by cue duration condition, separately. In the $150 \mathrm{~ms}$ SOA, differences between valid RTs and invalid RTs were not significant in the 50 and $100 \mathrm{~ms}$ cue durations, but they were significant for the $200 \mathrm{~ms}$ cue duration $\left[F(1,112)=3.96, p<0.05, \eta_{\mathrm{p}}^{2}=0.03\right]$ and for the $300 \mathrm{~ms}$ cue duration $[F(1,112)=10.43, p<0.01$, $\left.\eta_{\mathrm{p}}^{2}=0.09\right]$. These results indicated that the overlap cues could induce facilitation but non-overlap cues could not. In the 450 ms SOA when IOR was expected, all the comparisons indicated significant differences between valid RTs and invalid RTs for the $50 \mathrm{~ms}$ cue duration $\left[F(1,112)=73.79, p<0.001, \eta_{\mathrm{p}}^{2}=\right.$ $0.39]$, for the $100 \mathrm{~ms}$ cue duration $[F(1,112)=60.19, p<0.001$, $\left.\eta_{\mathrm{p}}^{2}=0.35\right]$, for the $200 \mathrm{~ms}$ cue duration $[F(1,112)=34.23, p<$ $\left.0.01, \eta_{\mathrm{p}}^{2}=0.23\right]$, and for the $300 \mathrm{~ms}$ cue duration $[F(1,112)=$ $\left.30.93, p<0.001, \eta_{\mathrm{p}}^{2}=0.22\right]$, with slower RTs for the valid condition relative to those of the invalid condition.

Correlations between the facilitation effect and IOR. Correlations between facilitation and IOR were calculated (Table 1). The magnitude of the facilitation effect in the non-overlap cue condition correlated with that in the overlap cue condition ( $r=$ $0.60, p<0.01$ ). The magnitude of IOR in the non-overlap cue condition also correlated with that in the overlap cue condition $(r=0.45, p<0.05)$. These results indicated that individuals who showed larger facilitation or IOR in the non-overlap cue condition also showed larger effects in the overlap cue condition. In contrast, the correlations between the magnitude of the facilitation effect and that of IOR in the non-overlap cue condition nor
Table 1.

Correlations between facilitation effect and IOR for each condition of the cue and SOA.

\begin{tabular}{lcccc}
\hline & 1 & 2 & 3 & 4 \\
\hline 1) Non-overlap cue, $150 \mathrm{~ms} \mathrm{SOA}$ & - & & & \\
2) Non-overlap cue, $450 \mathrm{~ms} \mathrm{SOA}$ & .30 & - & & \\
3) Overlap cue, $150 \mathrm{~ms} \mathrm{SOA}$ & $.60^{*}$ & .19 & - & \\
4) Overlap cue, $450 \mathrm{~ms} \mathrm{SOA}$ & .20 & $.45^{*}$ & .11 & - \\
\hline
\end{tabular}

those in the overlap cue condition did not reach significance. The lack of significant correlations did not support the conventional idea that the facilitation effect and IOR were driven by the same mechanism.

Comparisons of IOR with the overlap cue and the non-overlap cue. Within-subjects $t$ tests were performed on the magnitude of IOR of the cue duration conditions. It revealed that the IORs of the two cue durations were comparable in magnitude regardless the magnitude of the facilitation effect $[t(30)=1.87$, $p>0.07, d=0.02]$.

Comparisons of two IOR groups formed by the magnitude of the facilitation effect. Top twelve participants who showed larger magnitude of the facilitation effect were assigned to the facilitation group. Twelve other participants who showed smalller facilitation were grouped as non-facilitation group. The classifications were done separately for each cue duration condition. Independent-sample $t$ tests were performed on the magnitude of IOR (Figure 4). In the non-overlap cue condition, the magnitude of IOR of the two facilitation groups were comparable $[t(38)=1.72, p>.33, d=0.41]$. This was also the case for the overlap cue condition $[t(38)=1.72, p>.69, d=0.16]$. Again, the lack of significant differences indicated that IOR and the facilitation effect were not controlled by the same mechanism.

\section{General Discussion}

The first aim of this study was to shed light on the question of how the cue duration affects the facilitation effect and IOR. The type of the cue used in the studies of Maruff et al. (1999) 


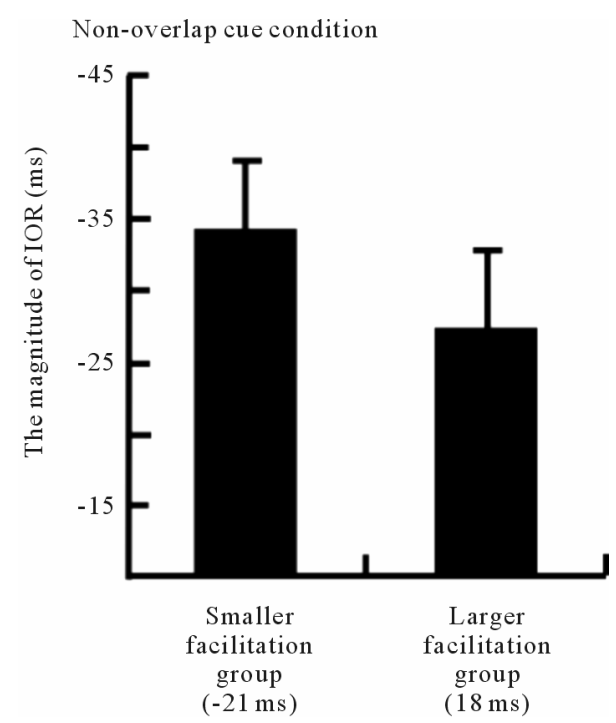

(a)

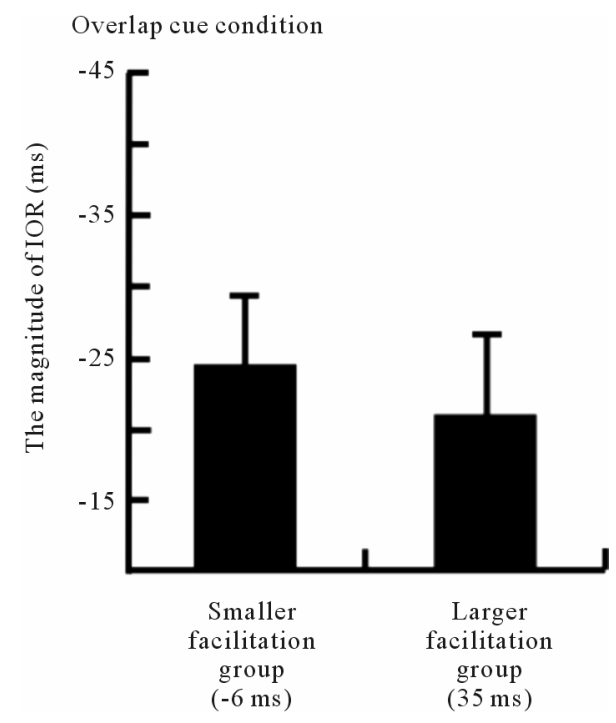

(b)

Figure 4.

Mean magnitude of IOR of larger and smaller facilitation group. The panel (a) showed the results of comparison with the non-overlap cue. The panel (b) showed the results of comparison with the overlap cue. The magnitude of IOR was calculated by invalid RTs minus valid RTs. Error bars indicate standard error.

and Collie et al. (2000) might have led to their findings that these two attentional effects were correlated because their cue was presented as a new object with an abrupt onset, rather than more conventional type of the cue of incremental brightness change used in majority of the spatial cueing studies (e.g. Berger et al., 1999). In this study, we used the latter type of more conventional cueing procedure (i.e., brightening of one of two boxes) with a small dot as the target. In Experiments 1 and 2, the overlap cue produced the facilitation effect when the SOA was $150 \mathrm{~ms}$. In contrast, the non-overlap cue did not produce significant facilitation for the same SOA condition. These results suggest that the temporal overlap of the cue was necessary in producing the facilitation effect. Importantly, the attentional capture effect of the temporal property of the cue was dependent on the cue-target temporal relationship (overlap or nonoverlap) rather than on the cue duration per se, as shown in Experiment 2. Overall, in terms of the cue duration effect on facilitation, the results in this study were congruent with the results reported in Maruff et al. (1999) and Collie et al. (2000) and incongruent with the results of Berger et al. (1999).

One explanation for the facilitation observed only in the overlap cue condition was that the overlap cue could capture attention more strongly than the non-overlap cue. This attentional account was well documented in the previous studies, although it is still possible to argue that sensory (energy) summation was partly responsible for the present results (Tassinari \& Berlucchi, 1992; Wright \& Richard, 2003). Although it is not easy to dismiss this possibility, we do not think that sensory summation was responsible for the present results because the cue and the target used in this study were not close enough to produce sensory summation and they were sufficiently different in their shapes. In this connection, Collie et al. (2000) examined in their Experiment 2 whether the facilitation effect spread from the cued placeholder at $18^{\circ}$ to the nearby placeholder at $9^{\circ}$. They used four placeholders that were arranged horizontally symmetrically across fixation point and the target was presented at one of these four placeholders. If it was due to energy summation, the facilitation effect should have been found to a restricted area around the cued location and would not have spread to wider area. Contrarily to this expectation, when the cue was presented at $18^{\circ}$, the facilitation effect was found not only at the cued location but also at the nearby location (i.e., the placeholder at $9^{\circ}$ ) as well. This wider spread of the facilitation effect could not be accounted for by sensory summation. Thus it seems that the facilitation effect arose by some attentional process rather than as a peripheral sensory effect.

The second aim of this study was to shed light on the relationship between IOR and attentional mechanism. There were four findings that suggested discrepancies in the effect of spatial cueing between the facilitation effect and IOR. First, IOR were observed with the 50 or $100 \mathrm{~ms}$ cue duration in the 450 and $800 \mathrm{~ms}$ SOA, while the facilitation effect did not observed in the $150 \mathrm{~ms}$ SOA. In contrast, when the cue duration was longer (i.e., 200 or $300 \mathrm{~ms}$ ), the facilitation effect and IOR were observed. These results indicated that temporal property of the cue affected only the facilitation effect, but not IOR. Second, there was no significant correlation between these two attentional effects in both the overlap and the non-overlap cue conditions. Third, the magnitude of IOR was equivalent irrespective of the cue durations. In Experiments 1 and 2, the facilitation effect was limited to the overlap cue condition, while comparable IORs were found for the overlap and non-overlap cue condition. If the facilitation effect and IOR were two aspects of the same attentional mechanism, when the non-overlap cue was not strong enough to drive the facilitation effect, it would not be observed as well with the result that the magnitude of IOR. Contrary to this expectation, IORs were observed both cue condition and did not differ from each other. Forth, individuals who showed larger facilitation did not show larger IOR either in the non-overlap cue condition or in the overlap cue condition. Overall, the results indicated that these two attentional phenomena were driven by two separate mechanisms.

In some cases, IOR is thought to be an indicator of previous location of attentional orienting. It is somewhat harder to obtain 
the facilitation effect as it is a fragile and short-lived phenomenon. In contrast, IOR is much more robust and long-lived phenomenon caused by the exogenous cue. Therefore, if the facilitation effect and IOR were driven by same mechanism, IOR might be used as a better indicator of the location of attention. However, if these two phenomena reflect distinct attentional mechanisms, it is not appropriate to use IOR as a sure indicator of previous location of attentional orienting. Thus, it is important to ascertain whether the facilitation effect and IOR were controlled by one and the same attentional mechanism or not. The results in this study added further evidence for the independence of these two phenomena that reflected the automatic control of attention. The results in this study support the notion that the facilitation effect and IOR are influenced by separate factors (both stimulus properties and participants' traits) and presumably are controlled by different attentional mechanisms at least in the spatial cueing method. It follows that IOR cannot be regarded as an indicator of attentional capture.

\section{Acknowledgements}

The study was partially supported by The Grants-in-Aid for Scientific Research (No. 24530908) to the second author.

\section{REFERENCES}

Berger, A., Dori, H., \& Henik, A. (1999). Peripheral non-informative cues do induce early facilitation of target detection. European Journal of Cognitive Psychology, 11, 119-137. doi:10.1080/713752304

Berlucchi. (2006). Inhibition of return: A phenomonon in search of a mechanism and a better name. Cognitive Neuropsychology, 23, 10651074. doi:10.1080/02643290600588426

Collie, A., Maruff, P., Yucel, M., Danckert, J., \& Currie, J. (2000). Spatiotemporal distribution of facilitation and inhibition of return arising from the reflexive orienting of covert attention. Journal of Experimental Psychology: Human Perception and Performance, 26, 1733-1745. doi:10.1037/0096-1523.26.6.1733

Gibson, B. S., \& Egeth, H. (1994). Inhibition and disinhibition of return: Evidence from temporal order judgments. Perception \& Psychophysics, 56, 669-680. doi:10.3758/BF03208360

Jonides, J., \& Yantis, S. (1988). Uniqueness of abrupt onset in capturing attention. Perception \& Psychophysics, 43, 346-354. doi:10.3758/BF03208805

Klein, R. M. (2000). Inhibition of return. Trends in Cognitive Science, 4, 138-147. doi:10.1016/S1364-6613(00)01452-2

Maruff, P., Yucel, M., Danckert, J., Stuart, G., \& Currie, J. (1999).
Facilitation and inhibition arising from the exogenous orienting of covert attention depends on the temporal properties of spatial cues and targets. Neuropsychologia, 37, 731-744. doi:10.1016/S0028-3932(98)00067-0

Maylor, E. A. (1985). Facilitatory and inhibitory components of orienting in visual space. In M. I. Posner, \& O. S. M. Martin (Eds.), Attention and Performance XI (pp. 184-204). Hillsdale, NJ: Erlbaum.

McAuliffe, J., \& Pratt, J. (2005). The role of temporal and spatial factors in the covert orienting of visual attention tasks. Psychological Research, 69, 285-291. doi:10.1007/s00426-004-0179-4

O'Donnell, C., \& Pratt, J. (1996). Inhibition of return along the path of attention. Canadian Journal of Experiment Psychology, 50, 386-392. doi:10.1037/1196-1961.50.4.386

Posner, M. I. (1978). Chronometric explorations of mind. Hillsdale, NJ: Erlbaum.

Posner, M. I. (1980). Orienting of attention. Quarterly Journal of Experimental Psychology, 32, 3-25. doi:10.1080/00335558008248231

Posner, M. I., \& Cohen, Y. (1984). Components of attention. In H. Bouma, \& D. Bowhuis (Eds.), Attention and Performance $X$ (pp. 531-556). Hillsdale, NJ: Erlbaum.

Posner, M. I., Rafal, R. D., Choate, L., \& Vaughan, J. (1985). Inhibition of return: Neural basis and function. Cognitive Neuropsychology, 2, 211-228. doi: $10.1080 / 02643298508252866$

Pratt, J., Hillis, J., \& Gold, J. M. (2001). The effect of the physical characteristics of cues and targets on facilitation and inhibition. Psychonomic Bulletin \& Review, 8, 489-495. doi:10.3758/BF03196183

Rafal, R. D., Calabresi, P. A., Brennan. C. W., \& Sciolto, T. K. (1989). Saccade preparation inhibits reorienting to recently attended locations. Journal of Experimental Psychology: Human Perception and Performance, 15, 673-685. doi:10.1037/0096-1523.15.4.673

Reuter-Lorenz, P. A., Jha, A. P., \& Rosenquist, J. N. (1996). What is inhibited in inhibition of return? Journal of Experimental Psychology: Human Perception \& Performance, 22, 367-378. doi:10.1037/0096-1523.22.2.367

Tassinari, G., \& Berlucchi, G. (1992). Sensory and attentional components of slowing of manual reaction time to non-fixated visual targets by ipsilateral primes. Vision Research, 33, 1525-1534. doi:10.1016/0042-6989(93)90145-M

Taylor, T. L., \& Klein R. M. (1998). On the causes and effects of inhibition of return. Psychonomic Bulletin \& Review, 5, 625-643. doi:10.3758/BF03208839

Wright, R. D., \& Richard, C. M. (2003). Sensory mediation of stimulus-driven attentional capture in multiple-cue displays. Perception \& Psychophysics, 65, 925-938. doi:10.3758/BF03194824

Yantis, S., \& Jonides, J. (1984). Abrupt visual onsets and selective attention: Evidence from visual search. Journal of Experimental Psychology: Human Perception \& Performance, 10, 601-621. doi:10.1037/0096-1523.10.5.601. 